\title{
Registro de Leopardus tigrinus (Carnivora: Felidae) y Cuniculus taczanowskii (Rodentia: Cuniculidae) en fragmentos de bosque nativo en el municipio de Copacabana (Antioquia)
}

\author{
Julio Montoya-Osorio ${ }^{12 *}$ (iD), Alejandro Arango-Lopera² (iD) \\ 1 Secretaría de Educación del Municipio de Medellín. \\ 2 Grupo de Investigación y Observación Ambiental, carrera 48 \# 50 -38 Apto 302, Copacabana, Colombia. \\ *Correspondencia: juliomolt@gmail.com
}

\section{Resumen}

Esta nota presenta el registro de la Oncilla (Leopardus tigrinus) y la Guagua de Montaña (Cuniculus taczanowskii) en Copacabana, municipio de Antioquia (Colombia). Estos registros se realizaron en los años 2018 y 2019 con cámaras trampa en la vertiente noroccidental del municipio, en fragmentos de bosques de montaña con predominio de flora nativa. Se obtuvieron cuatro registros para Cuniculus taczanowskii, y un solo registro para Leopardus tigrinus. La presencia de ambas especies en los lugares monitoreados sugiere que estos bosques brindan los recursos para su subsistencia. Es importante promover la conectividad ecológica de dichos fragmentos para contribuir en la supervivencia de las especies que allí habitan.

Palabras clave: cámaras trampa, conservación, Valle de Aburrá.

\begin{abstract}
This note shows the register of the Oncilla (Leopardus tigrinus) and the Mountain Paca (Cuniculus taczanowskii) in Copacabana, a municipality in Antioquia (Colombia). These records were taken in 2018 and 2019 with camera trapping in the northwestern slope of the municipality, in fragments of mountain forests with a predominance of native flora. Four records were obtained for Cuniculus taczanowskii, and a single record for Leopardus tigrinus. The presence of both species in the monitored places suggests that these forests provide the resources for their subsistence. It is important to promote the ecological connectivity of these fragments to contribute to the survival of the species that inhabit there.
\end{abstract}

Key words: camera traps, conservation, Aburrá Valley 
El Valle de Aburrá es una región ubicada en el norte de la cordillera central de los Andes, en el departamento de Antioquia, donde se encuentra una alta densidad poblacional humana en el territorio (Agudelo 2010). El Valle está formado por una depresión del relieve en cuyo fondo encontramos una altura mínima de 1400 msnm, que limita dos laderas que alcanza una altura máxima de 3000 msnm (Aristizábal \& Yokota 2008). La vertiente occidental y oriental se encuentran separadas por el entorno urbano de los municipios. En la parte alta de las laderas, se encuentran fragmentos de bosques nativos que persisten a pesar de la presión antrópica generada por la ampliación del uso del suelo urbano, agrícola y ganadero (Vásquez Peinado \& González-Caro 2018). Gracias al esfuerzo realizado por diferentes personas y organizaciones, se han realizado registros de mamíferos en el territorio, entre los que podemos mencionar los trabajos de Allen 1916; Cuartas-Calle \& Muñoz-Arango 2003; Delgado 2007, 2009; Delgado et al. 2011; Arias-Alzate et al. 2013; AriasAlzate et al. 2014; Sánchez-Londoño et al. 2014; Arias-Alzate et al. 2015; Arias-Alzate et al. 2016; Quintana Diosa et al. 2016; Bedoya-V et al. 2018; Sánchez-Londoño et al. 2019; VilladaCadavid \& Soto-Calderón 2020.

Dentro de la fauna registrada en las laderas del Valle de Aburrá, se encuentran la Oncilla (Leopardus tigrinus Schreber, 1775), reportada por atropellamiento en la vía, así como con cámaras trampa (Delgado 2007; Arias-Alzate et al. 2014; Quintana Diosa et al. 2016; SánchezLondoño et al. 2019), y la Guagua de Montaña (Cuniculus taczanowskii Stolzmann, 1865), registrada por medio de cámaras trampa (Delgado-V et al. 2011; Sánchez-Londoño et al. 2019). Sin embargo, poco se conoce de la presencia de mamíferos en el norte del Valle de Aburrá, donde existe una fuerte presión sobre los ecosistemas, con pocos procesos de conservación y restauración, lo que lleva a un desconocimiento general de la distribución de las especies, su abundancia poblacional, estado de conservación y los servicios ambientales que prestan (Sánchez-Londoño et al. 2014).

Con el fin de confirmar la distribución de mamíferos en la ladera noroccidental del municipio de Copacabana, se eligieron dos fragmentos de bosque nativo con abundante cobertura vegetal, el primero de 112 ha en la vereda La Veta y el segundo de 104 ha en la vereda Zarzal la Luz, para realizar seguimiento con cámaras trampa (Figura 1). Estas se ubicaron dentro del bosque en lugares que se consideraban corredores de fauna y en las márgenes de la zona boscosa, utilizando como atrayente sardina, esencia de vainilla, avena y mantequilla de maní. Las cámaras fueron ubicadas (una en cada fragmento), entre el 1418 de abril, 9-24 de junio del 2018, 13-27 de octubre, 3-17 de noviembre y 7-21 de diciembre del 2019, alcanzando un esfuerzo de muestreo de 150 cámaras/día con dos cámaras Bushnell Trophy con resolución de $24 \mathrm{MP}$, programadas para funcionamiento de video y fotografía con sensor durante el día y la noche. Las cámaras se colocaron entre 40 y $50 \mathrm{~cm}$ del suelo. En ambos fragmentos se realiza un recorrido preliminar para identificar las especies vegetales de importancia en el sector, de lo que se puede mencionar una predominancia en el dosel de las especies Roble de Tierra Fría (Quercus humboldtii Bonpl.), Carate (Vismia laevis Triana y Planch) y Manzanillo (Rhus striata Ruiz \& Pav.), con un sotobosque abundante en especies como el Anturio (Anthurium yarumalense Engl.), helechos (de los géneros Sphaeradenia, Campyloneurum y Thelypteris), Chusque (Chusquea sp.) y Espino Cruceto (Duranta mutisii L. f.).

Con un esfuerzo de muestreo de 150 cámaras/día, se obtuvo la evidencia visual de la presencia de las especies Leopardus tigrinus y Cuniculus taczanowskii (Figura 2) para el municipio de Copacabana en el norte del Valle de Aburrá, que se resume en la tabla 1. 


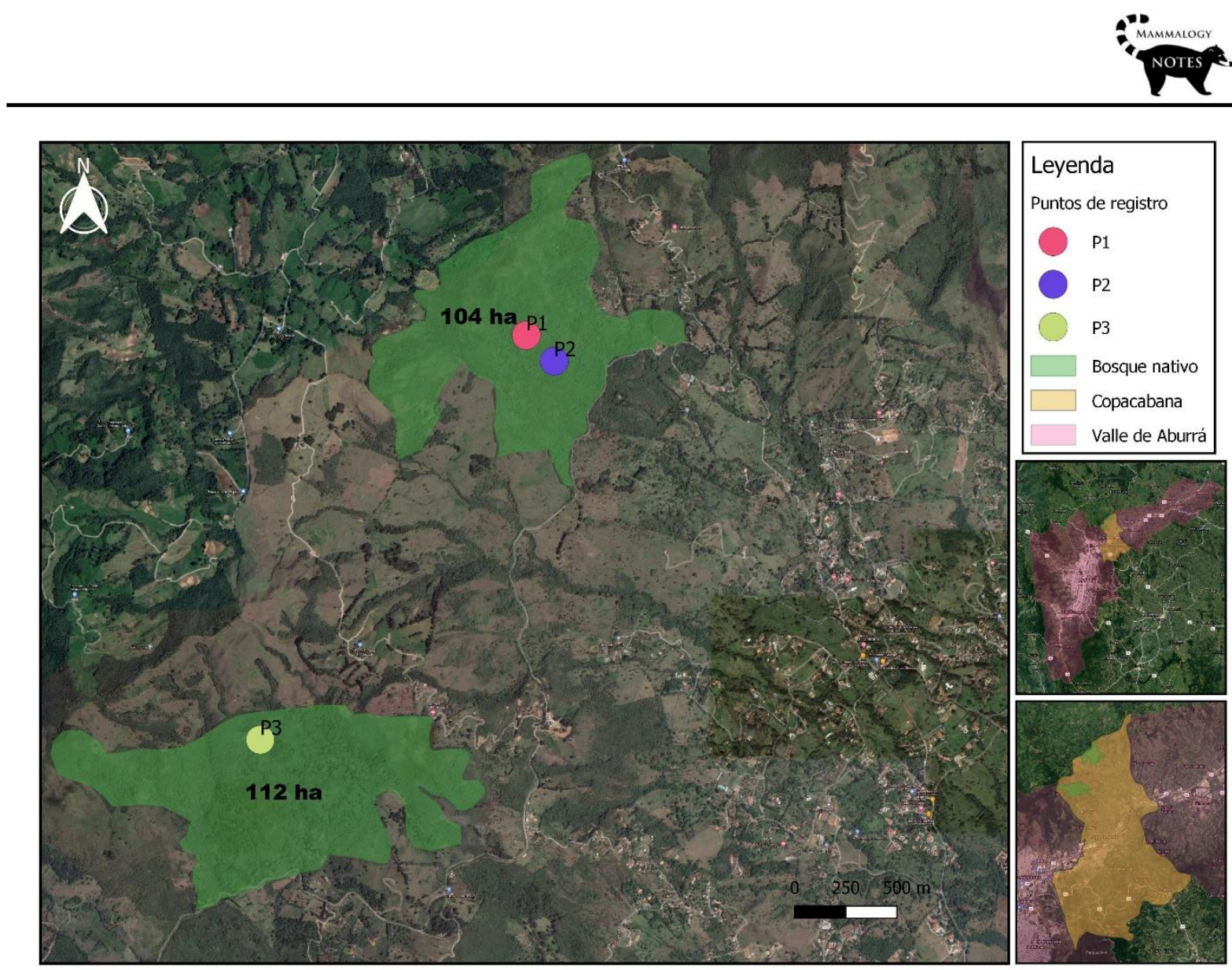

FIGURA 1. Área de estudio. En el P1 se registra un individuo de Leopardus tigrinus, en P1, P2 y P3 se registra individuos de Cuniculus taczanowskii

Las especies registradas se encuentran en amenaza de extinción según la IUCN: La Oncilla (Leopardus tigrinus) se clasifica en estado vulnerable (Payan \& de Oliveira. 2016) y la Guagua de Montaña (Cuniculus taczanowskii) como casi amenazada (Roach 2016). La pérdida y transformación de los hábitats naturales por el avance de la frontera agropecuaria, la fragmentación de sus poblaciones por carreteras y poblados y la cacería ilegal afectan las poblaciones naturales de ambas especies (Payan \& González-Maya 2011; Payán \& Soto 2012; González-Maya et al. 2012; Sierra-Giraldo \& Escobar-Lasso 2014; Payan \& De Oliveira 2016; Roach 2016). Otra de las amenazas sobre las poblaciones de mamíferos en el Valle de Aburrá, es el atropellamiento en carreteras y caminos (Delgado 2007; AriasAlzate et al. 2014; Bedoya-V et al. 2018).

TABLA 1. Resumen de los registros obtenidos con las cámaras trampa.

\begin{tabular}{lllll}
\hline Especie & Fecha & Punto & $\begin{array}{l}\text { coordenadas } \\
\text { WGS84 }\end{array}$ & $\begin{array}{l}\text { Altura } \\
(\mathrm{msnm})\end{array}$ \\
\hline Leopardus tigrinus & $11 / 12 / 2019$ & P1 & $6.405770,-75.511340$ & 2.322 \\
& $10 / 06 / 2018$ & P2 & $6.404618,-75.510092$ & 2.218 \\
Cuniculus taczanowskii & $13 / 06 / 2018$ & P2 & $6.404618,-75.510092$ & 2.218 \\
& $14 / 10 / 2019$ & P3 & $6.387971,-75.523122$ & 2.506 \\
\hline
\end{tabular}

Existen publicaciones de registros para la presencia de ambas especies, en su mayoría para la ladera oriental del sur del Valle de Aburrá en los municipios de Medellín, Envigado y Caldas (Delgado 2007; Delgado-V et al. 2011; Arias-Alzate et al. 2014; Quintana Diosa et al. 


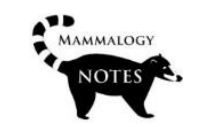

2016; Sánchez-Londoño et al. 2019), siendo este el primer registro para el municipio de Copacabana en el norte del Valle de Aburrá.
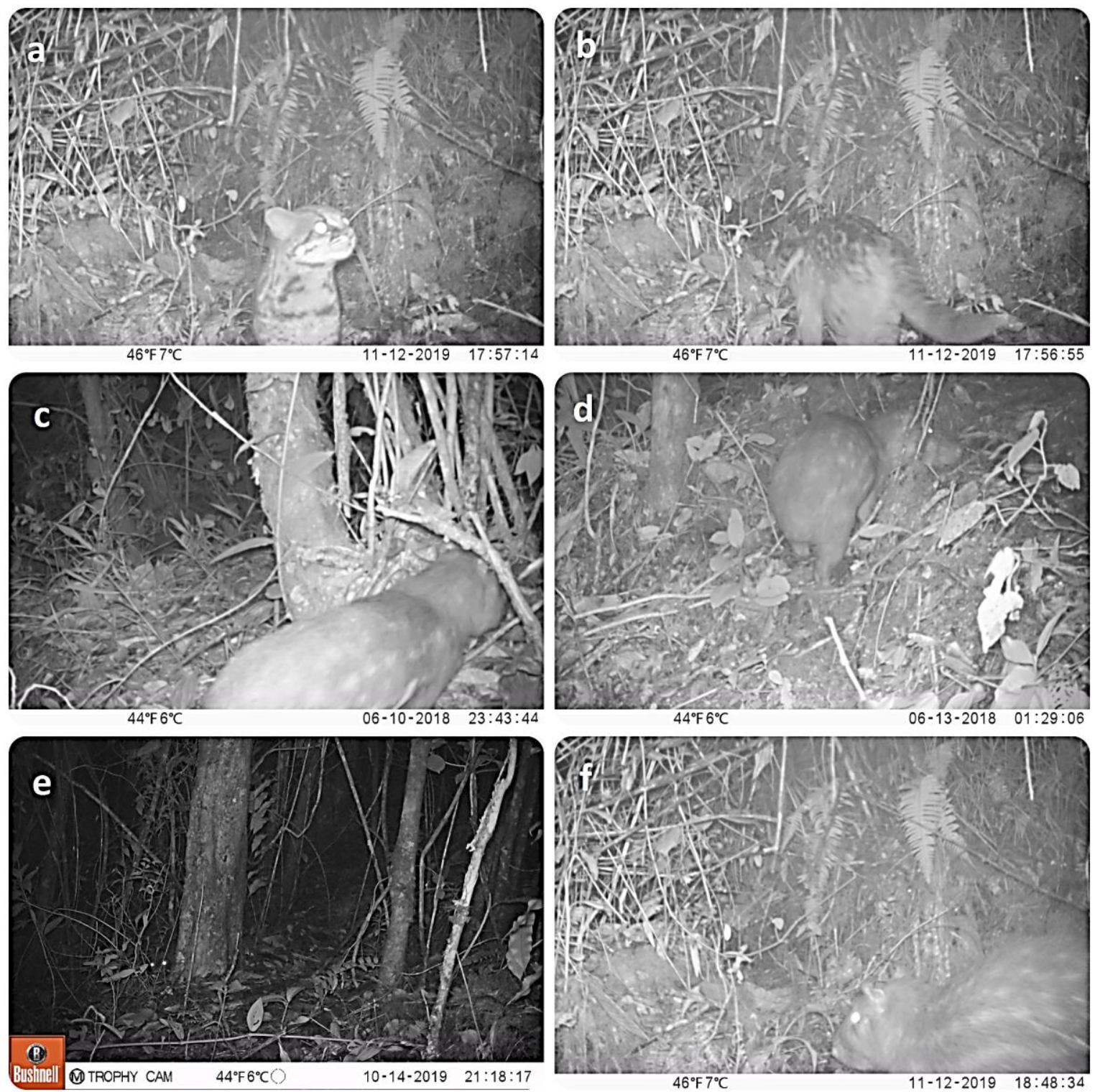

FIGURA 2. Registros obtenidos con cámara trampa para Leopardus tigrinus (a, b) y Cuniculus taczanowskii (c, d, e, f) en el municipio de Copacabana en el departamento de Antioquia.

El encontrar ambas especies en los lugares monitoreados, sugiere que estos bosques brindan los recursos para su subsistencia, a pesar del alto grado de fragmentación, lo que suscita la necesidad de promover la conectividad ecológica de dichos fragmentos para contribuir en la supervivencia de las especies que allí habitan. La alta intervención antrópica de estos hábitats, limita la supervivencia de estas especies, así como disminuye la calidad de los bienes y servicios que brindan dichos bosques al ser humano, por lo que se recomienda que estos lugares sean tenidos en cuenta en los proyectos de conservación regional y municipal. Estos hallazgos demuestran la importancia de los estudios y monitoreos de la diversidad biológica, para promover estrategias de conservación, protección y restauración de los ecosistemas naturales. 


\section{AGRADECIMIENTOS}

Este trabajo ha sido fruto de la dedicación y compromiso del grupo de trabajo de la Corporación Grupo de Investigación y Observación Ambiental (GIO Ambiental) y su interés de reconocer el estado de los ecosistemas del Valle de Aburrá y el departamento de Antioquia para diseñar estrategias educativas con el fin de promover el cuidado de los ecosistemas naturales. Se agradece a todas las personas que contribuyeron de una u otra manera a la realización de este documento.

\section{REFERENCIAS}

Agudelo PL. 2010. La ciudad sostenible. Dependencia ecológica y relaciones regionales. Un estudio de caso en el área metropolitana de Medellín, Colombia. Universidad Nacional de Colombia. Medellín. 196 p.

Allen JA. 1916. List of mammals collected in Colombia by the American Museum of Natural History expeditions, 1910-1915. Bulletin of American Museum of Natural History, 35:191-238

Arias-Alzate A, Delgado-V CA, Ortega JC, Botero-Cañola S, Sánchez-Londoño JD. 2013. Presencia de Puma yagouaroundi (Carnivora: Felidae) en el valle de Aburrá, Antioquia, Colombia. Brenesia. 79 83-84.

Arias-Alzate A, Sánchez-Londoño JD, Botero-Cañola S, González-Maya JF. 2014. Registros confirmados recientes de la Oncilla (Leopardus tigrinus) en el departamento de Antioquia, Colombia. Mammalogy Notes, 1(2), 4-5. https:/ / doi.org/10.47603/manovol1n2.4-5

Arias-Alzate A, Delgado-V CA, Navarro JF, González-Maya JF. 2015. Presencia de Puma (Puma concolor) en un paisaje periurbano al sur del Valle de Aburrá, Antioquia, Colombia. Mammalogy Notes, 2(1), 24-28. https: / / doi.org/10.47603/manovol2n1.24-28

Arias-Alzate A, Delgado-V CA, Navarro JF. 2016. Nuevos registros de simpatría de Nasua nasua y Nasuella olivacea (Carnivora: Procyonidae) en el Valle de Aburrá (Antioquia) y anotaciones sobre sus distribuciones en Colombia. Mammalogy Notes, 3 (1-2), 49-53. https://doi.org/10.47603/manovol3n1.49-53

Aristizabal E, Yokota S. 2008. Evolución geomorfológica del Valle de Aburrá y sus implicaciones en la ocurrencia de movimientos en masa. Boletín de Ciencias de la Tierra: 24 (julio), 5-18.

Bedoya-V MM, Arias-Alzate A, Delgado-V CA. 2018. Atropellamientos de fauna silvestre en la red vial urbana de cinco ciudades del Valle de Aburrá (Antioquia, Colombia). Caldasia 40(2), 335348. Julio-diciembre 2018. https:// doi.org/10.15446/caldasia.v40n2.68297

Cuartas-Calle CA, Muñoz-Arango J. 2003. Lista de los mamíferos (Mammalia: Theria) del departamento de Antioquia, Colombia. Biota Colombiana 4(1), 65-78.

Delgado-V CA. 2007. Muerte de mamíferos porvehículos en la vía del Escobero, Envigado (Antioquia), Colombia. Actualidades Biológicas, 29(87), 229-233.

Delgado-V CA. 2009. Non-volant mammals, Reserva San Sebastián-La Castellana, Valle de Aburrá, Antioquia, Colombia. Check list, 5(1), 1-4. https:// doi.org/10.15560/5.1.1

Delgado-V CA, Arias-Alzate A, Botero S, Sánchez-Londoño JD. 2011. Registro de Cuniculus taczanowskii (Rodentia: Cuniculidae) y Eira barbara (Carnivora: Mustelidae) en una zona periurbana de Medellín, Colombia. Brenesia. 75-76, 124-126.

Delgado-V CA, Arias-Alzate A, Botero S, Sánchez-Londoño JD. 2011. Behaviour of the Tayra Eira Barbara near Medellín, Colombia: preliminary data from a video capturing survey. Small Carnivore Conservation, 44, 19-21. 
González-Maya JF, Cepeda A, Zárrate-Charry D, Granados-Peña R, Pérez Ascanio W, González M. 2012. Conflictos felinos-vida silvestre en el Caribe Colombiano: un estudio de caso en los departamentos del Cesar y La Guajira. En el Plan de Conservación de Felinos del Caribe colombiano: Los felinos y su papel en la planificación regional integral basada en especies clave. Castaño-Uribe C, González-Maya JF, Zárrate-Charry D, Ange-Jaramillo C, Vela-Vargas IM. (Eds). Fundación Herencia Ambiental Caribe, ProCAT Colombia, The Sierra to Sea Institute. Santa Marta, Colombia, pp. 48-57.

Payan E, González-Maya JF. 2011. Distribución geográfica de la Oncilla (Leopardus tigrinus) en Colombia e implicaciones para su conservación. Revista Latinoamericana de Conservación, 2, 51-59.

Payan E, Soto VC. 2012. Los Felinos de Colombia. Ministerio de Ambiente y Desarrollo Sostenible, Instituto de Investigaciones de Recursos Biológicos Alexander von Humboldt y Panthera Colombia. 48 pp.

Payan E, De Oliveira, T. 2016. Leopardus tigrinus. The IUCN Red List of Threatened Species 2016. Versión en línea. 22 de marzo del 2021. https://dx.doi.org/10.2305/IUCN.UK.20162.RLTS.T54012637A50653881.en

Quintana LE, Carmona M, Plese T, David CA, Monsalve S. 2016. Análisis de la biodiversidad de fauna vertebrada en una finca de Caldas, Antioquia. Rev Med Vet (32), 53-65. https:// doi.org/10.19052/mv.3855

Roach N. 2016. Cuniculus taczanowskii. The IUCN Red List of Threatened Species 2016. 22 de marzo del 2021. https:// dx.doi.org/10.2305/IUCN.UK.2016-2.RLTS.T700A22197554.en

Sánchez-Londoño JD, Marín-C D, Botero-Cañola S, Solari S. 2014. Mamíferos Silvestres del Valle de Aburrá. App no activa. Área Metropolitana del Valle de Aburrá, Corantioquia, Universidad de Antioquia. Medellín.

Sánchez-Londoño JD, Gómez-R DA, Solari S, Molina A. 2019. Mamíferos (Mammalia) de la Reserva Forestal Protectora Alto de San Miguel (Caldas-Antioquia, Colombia). En: Sánchez-Londoño JD, Tuberquia DJ, Parra JL, editores. Estudios en Biodiversidad del Alto de San Miguel. Medellín, Colombia: Editorial Universidad CES. 69-89.

Sierra-Giraldo J, Escobar-Lasso S. 2014. Field observations on hunting of Dinomys branickii and Cuniculus taczanowskii (Mammalia: Rodentia) within the Sub-Andean forest fragments of the Colombian Central Cordillera. Bioma, 2. 21-34.

Vásquez PA \& González-Caro S. 2018. Estado de los bosques de Antioquia entre 1990-2015. 63-80. En Quintero Vallejo E, Benavides AM, Moreno N, González-Caro S. (Ed.), Bosques Andinos, estado actual y retos para su conservación en Antioquia. Medellín, Colombia: Fundación Jardín Botánico de Medellín Joaquín Antonio Uribe. Programa Bosques Andinos (COSUDE). 1 EdMedellín, 2018, 542 páginas.

Villada-Cadavid T, Soto-Calderón ID. 2020. Diversidad de mamíferos en un remanente de bosque urbano de la ciudad de Medellín (Antioquia, Colombia). Actualidades biológicas, 42(113), 111. https://doi.org/10.17533/udea.acbi.v42n113a05

Editor: José F. González-Maya

Recibido: 2020-03-26

Revisado: 2020-04-15

Aceptado: 2020-04-28

Publicado: 2021-06-04 